\title{
Detection and Analysis of Tremor by Inertial Sensing Method
}

\author{
Niranjan K.R, Beena Ullala Mata B N and Girish
}

\begin{abstract}
This paper proposes the prototype of the technology to capture the movement data at the point of interest related to the human body. The movement data is collected from a range of supported system using inertial sensor. The proposed system is built with an algorithm to remove the noise and to extract the intent data obtained from the samples after the data processing. This work contributes the novelty in developing a inertial assessment device which enables on-sensor-node data processing. The benefit of this device is its smaller size and light in weight.
\end{abstract}

Keywords--- Cerebellar Tremor, Musculo-skeletal System, MEMS, Passive Infrared Sensor, Pill-rolling, Raw Inertialdata, Stimulants, Thalamotomy

\section{INTRODUCTION}

\section{A. Introduction to Tremors}

Tremor is defined as a swift back-and-forth movement of a body segment. It is one of the most common disorder come across in clinical practice and in most of the instances it is one of the phenomenon related to motor [1].

A tremor activity involves the movements (oscillations or twitching) of one or more body parts with respect to muscle contraction and relaxation. It is an involuntary activity, which is somewhat periodic. It can affect the hands, arms, eyes, face, head, vocal folds, trunk, and legs. Most tremors occur in the hands. In some people, tremor is a sign of another neurological syndrome. A very common kind of tremor is the chattering of teeth, usually induced by cold temperatures or by fear. It is one of the most common unintentional movements.

\section{B. Causes of Tremor}

Tremor can be a sign related with disorders in those parts of the brain that control muscles through the body or in particular areas, such as the hands. Neurological disorders or conditions that can produce tremor includes multiple sclerosis, stroke, traumatic brain injury, chronic kidney disease and a number of neurodegenerative diseases that harm the parts of the brainstem or the cerebellum. Parkinson's disease is the one which is related to the tremor. Other causes includes the use of drugs, alcohol consumption, mercury poisoning. This also exists in infants with phenylketonuria (PKU), overexcited

Niranjan K.R, Assistant Professor, Medical Electronics, BMSCE, Bangalore, India.E-mail:niranjankr.ml@bmsce.ac.in

Beena Ullala Mata B N, Associate Professor, Medical Electronics, BMSCE, Bangalore, India.E-mail:bnb.ml@bmsce.ac.in

Girish, Engineer, Medical Electronics, BMSCE, Bangalore, India. Email:Giri12@gmail.com

DOI: 10.9756/BIJMMI.8067 thyroid or liver failure. Tremors can be an indication of hypoglycaemia, along with palpitations, sweating and anxiety. Tremor can also be caused due to dearth of sleep, dearth of vitamins, or increased stress. Lack of magnesium and thiamine have also been known to cause tremor or shaking, which resolves when the deficiency is corrected. Some forms of tremor are hereditarily transmitted in families, while others have no known cause. As one of the example tremors can also be caused by red-back spider which can be seen in Australia.

\section{Types of Tremor}

Tremor is most commonly classified by clinical features, cause or origin. Some of the better known forms of tremor, with their symptoms are given below:

1) Cerebellar tremor: Cerebellar tremor is a slow, broad tremor of the boundaries that occurs at the end of a focused movement, such as trying to press a button or touching a finger to the tip of one's nose. Cerebellar tremor is caused by abrasions in or injury to the cerebellum resulting from stroke, tumour, or disease such as multiple sclerosis or some hereditarily transmitted syndrome. It can also result from prolonged alcoholism or misuse of some medicines. In classic cerebellar tremor, a abrasion on one side of the brain produces a tremor in that same side of the body that go downhill with directed movement. Cerebellar damage can also produce a "wing-beating" type of tremor called rubral or Holmes' tremor - a combination of rest, action, and postural tremors. The tremor is often most noticeable when the affected person is active or is maintaining a particular posture. Cerebellar tremor may be accompanied by other manifestations of ataxia, including speech problems, involuntary eye movements, gait problems and postural tremor of the trunk and neck. Titubation is tremor of the head and is of cerebellar origin.

2) Essential tremor: is the most common type of tremor among the tremor types. Although the tremor may be mild and non-progressive in some people, in others, the tremor is gradually progressive, starting on one side of the body but affecting both sides within 3 years [2]. The hands are most commonly affected but the head, voice, tongue, legs, and trunk may also be involved.

Head tremor may be seen as a yes-yes or no-no motion. Vital tremor may be accompanied by mild gait disturbance. Tremor frequency may decrease as the person ages, but the severity may increase, affecting the person's ability to perform certain tasks or activities of daily living. Heightened feelings, 
stress, fever, physical exhaustion, or low blood sugar may cause tremors or increase their severity. Arrival is most common after age 40, although symptoms can appear at any age. It may occur in more than one family member. Children of a parent who has essential tremor have a 50 percent chance of inheriting the condition. Essential tremor is not associated with any known pathology.

3) Parkinsonian tremor: Parkinsonian tremor is caused by damage to structures within the brain that control movement. This resting tremor, which can occur as an isolated symptom or be seen in other disorders, is often a precursor to Parkinson's disease (more than 25 percent of patients with Parkinson's disease have an associated action tremor). The tremor, which is classically seen as a "pill-rolling" action of the hands that may also affect the chin, lips, legs, and trunk, can be markedly increased by stress or emotions. Onset of parkinsonian tremor is generally after age 60 . Movement starts in one limb or on one side of the body and usually progresses to include the other side [3].

4) Physiologic tremor: Physiologic tremor occurs in every normal individual and has no clinical significance. It is rarely visible and may be heightened by strong emotion (such as anxiety or fear), physical exhaustion, hypoglycaemia, hyperthyroidism, heavy metal poisoning, stimulants, alcohol withdrawal or fever. It can be seen in all voluntary muscle groups and can be detected by extending the arms and placing a piece of paper on top of the hands. Enhanced physiologic tremor is a strengthening of physiologic tremor to more visible levels. It is generally not caused by a neurological disease but by reaction to certain drugs, alcohol withdrawal, or medical conditions including an overactive thyroid and hypoglycaemia. It is usually reversible once the cause is corrected.

5) Psychogenic tremor: Psychogenic tremor (also called hysterical tremor) can occur at rest or during postural or kinetic movement. The characteristics of this kind of tremor may vary but generally include sudden onset and remission, increased incidence with stress, change in tremor direction and/or body part affected, and greatly decreased or disappearing tremor activity when the patient is distracted. Many patients with psychogenic tremor have a conversion disorder or another psychiatric disease.

6) Rubral tremor: Rubral tremor is characterized by coarse slow tremor which is present at rest, at posture and with intention. This tremor is associated with conditions which affect the red nucleus in the midbrain, classically unusual structure. Tremor may occur at any age but is most common in middle-age and older persons, typically around the age of 52-61. It may be occasional, temporary, or occur intermittently. Tremor affects men and women equally. The degree of tremor should be assessed in four positions. The tremor can then be classified by which position most accentuates the tremor. Table 1 shows the assessing positions of Tremor.

\section{Diagnosis}

During a physical exam a doctor can determine whether the tremor occurs primarily during action or at rest. The doctor will also check for tremor symmetry, any sensory loss, weakness or muscle atrophy, or decreased reflexes. A detailed family history may indicate if the tremor is inherited. Blood or urine tests can detect thyroid malfunction, other metabolic causes, and abnormal levels of certain chemicals that can cause tremor. These tests may also help to identify contributing causes, such as drug interaction, chronic alcoholism, or another condition or disease. Diagnostic imaging using CT or MRI imaging may help determine if the tremor is the result of a structural defect or degeneration of the brain.

Table 1: Assessing Positions of Tremor

\begin{tabular}{|l|l|l|}
\hline Position & Name & Description \\
\hline At rest & $\begin{array}{l}\text { Resting } \\
\text { tremors } \\
\text { including Parkinsonian syndromes } \\
\text { and essential tremor if severe. This } \\
\text { includes drug- induced tremors } \\
\text { from blockers of dopamine } \\
\text { receptors such as haloperidol and } \\
\text { other antipsychotic drugs. }\end{array}$ \\
\hline $\begin{array}{l}\text { During } \\
\text { contraction } \\
\text { (e.g. tight fist } \\
\text { while the arm is } \\
\text { rested and } \\
\text { supported) }\end{array}$ & $\begin{array}{l}\text { Contraction } \\
\text { tremors }\end{array}$ & $\begin{array}{l}\text { Tremors those are worse during } \\
\text { supported contraction including } \\
\text { essential tremors and also } \\
\text { cerebellar and exaggerated } \\
\text { physiologic tremors such as } \\
\text { hyperadreenergic state or } \\
\text { hyperthyroidism. Drugs such as } \\
\text { adrenergic, anticholinergics and } \\
\text { xanthene's can exaggerate } \\
\text { physiologic tremor. }\end{array}$ \\
\hline $\begin{array}{l}\text { During posture } \\
\text { (e.g. with the } \\
\text { arms elevated } \\
\text { against gravity } \\
\text { as in 'bird- } \\
\text { wing' position) }\end{array}$ & $\begin{array}{l}\text { Posture } \\
\text { tremors }\end{array}$ & $\begin{array}{l}\text { Tremors that are worse with } \\
\text { posture including essential tremor } \\
\text { and exaggerated physiologic } \\
\text { tremor. }\end{array}$ \\
\hline $\begin{array}{l}\text { During } \\
\text { intention (e.g. } \\
\text { finger to nose } \\
\text { test) }\end{array}$ & $\begin{array}{l}\text { Intention } \\
\text { tremors }\end{array}$ & $\begin{array}{l}\text { Intention tremors are tremors that } \\
\text { are worse during intention e.g. as } \\
\text { the patient's hand approaches } \\
\text { target, including cerebellar } \\
\text { disorders. The terminology of } \\
\text { intention' is currently less used, to } \\
\text { the profit of 'kinetic'. }\end{array}$ \\
\hline
\end{tabular}

The doctor will perform a neurological examination to assess nerve function and motor and sensory skills. The tests are designed to determine any functional limitations, such as difficulty with handwriting or the ability to hold a utensil or cup. The patient may be asked to place a finger on the tip of her or his nose, draw a spiral, or perform other tasks or exercises.

The doctor may order an electromyogram to diagnose muscle or nerve problems. This test measures involuntary muscle activity and muscle response to nerve stimulation. The selection of the sensors used is important. In addition to 
studies of muscle activity, tremor can be assessed with accuracy using accelerometers.

\section{E. Treatment}

There is no cure for most tremors. The appropriate treatment depends on accurate diagnosis of the cause. Some tremors respond to treatment of the underlying condition. For example, in some cases of psychogenic tremor, treating the patient's underlying psychological problem may cause the tremor to disappear.

\section{F. Medications}

Medications remain the basis of therapy in many cases. Symptomatic drug therapy is available for several forms of tremor:

1) Parkinsonian tremor drug treatment involves L-DOPA and/or dopamine-like drugs such as pergolide, bromocriptine and ropinirole. These can be dangerous, however, as they may cause symptoms such as tardive dyskinesia, akathisia, clonus, and in rare instances tardive (late developing) psychosis. Other drugs used to lessen parkinsonian tremor include amantadine and anticholinergic drugs like benzatropine.

2) Essential tremor may be treated with beta blockers (such as propranolol and nadolol) or primidone, an anticonvulsant.

3) Cerebellar tremor typically does not respond to medical treatment.

4) Rubral tremor patients may receive some relief using L-DOPA or anticholinergic drugs. Surgery may be helpful.

5) Dystonic tremor may respond to diazepam, anticholinergic drugs, and intramuscular injections of botulinum toxin. Botulinum toxin is also prescribed to treat voice and head tremors and several movement disorders.

6) Primary orthostatic tremor sometimes is treated with a combination of diazepam and primidone. Gabapentin provides a relief in some cases.

7) Enhanced physiologic tremor is usually reversible once the cause is corrected. If symptomatic treatment is needed, beta blockers can be used.

\section{G. Lifestyle}

Eliminating tremor "triggers" such as caffeine and other stimulants from the diet is often recommended. Essential tremor may benefit from slight doses of ethanol, but the potential negative consequences of regular ethanol intake need to be taken into account. Physical therapy may help to reduce tremor and improve coordination and muscle control for some patients. A physical therapist will evaluate the patient for tremor positioning, muscle control, muscle strength, and functional skills. Teaching the patient to brace the affected limb during the tremor or to hold an affected arm close to the body is sometimes useful in gaining motion control. Coordination and balancing exercises may help some patients. Some therapists recommend the use of weights, splints, other adaptive equipment, and special plates and utensils for eating.

\section{H. Surgery}

Surgical intervention such as thalamotomy and deep brain stimulation may ease certain tremors. These surgeries are usually performed only when the tremor is severe and does not respond to drugs. Response can be excellent.

Thalamotomy, involving the creation of lesions in the brain region called the thalamus, is quite effective in treating patients with essential, cerebellar, or parkinsonian tremor. This in-hospital procedure is performed under local anaesthesia, with the patient awake. After the patient's head is secured in a metal frame, the surgeon maps the patient's brain to locate the thalamus. A small hole is drilled through the skull and a temperature-controlled electrode is inserted into the thalamus.

A low-frequency current is passed through the electrode to activate the tremor and to confirm proper placement. Once the site has been confirmed, the electrode is heated to create a temporary lesion. Testing is done to examine speech, language, coordination, and tremor activation, if any. If no problems occur, the probe is again heated to create a 3-mm permanent lesion. The probe, when cooled to body temperature, is withdrawn and the skull hole is covered. The lesion causes the tremor to permanently disappear without disrupting sensory or motor control.

Deep brain stimulation (DBS) uses implantable electrodes to send high-frequency electrical signals to the thalamus. The electrodes are implanted as described above. The patient uses a hand-held magnet to turn on and turn off a pulse generator that is surgically implanted under the skin. The electrical stimulation temporarily disables the tremor and can be "reversed," if necessary, by turning off the implanted electrode.

Batteries in the generator last about 5 years and can be replaced surgically. DBS is currently used to treat parkinsonian tremor and essential tremor. It is also applied successfully for other rare causes of tremor.

The most common side effects of tremor surgery include dysarthria (problems with motor control of speech), temporary or permanent cognitive impairment (including visual and learning difficulties), and problems with balance.

\section{Biomechanical Loading}

Apart from medication, rehabilitation programmes and surgical interventions, the application of biomechanical loading on tremor movement has been shown to be a technique that is able to suppress the effects of tremor on the human body. It has been established in the literature that most of the different types of tremor respond to biomechanical loading.In particular, it has been clinically tested that the increase of damping and/or inertia in the upper limb leads to a reduction of the tremors motion. Biomechanical loading relies on an external device that either passively or actively acts mechanically in parallel to the upper limb to counteract tremor movement. This phenomenon gives rise to the possibility of an orthotic management of tremor. 
Starting from this principle, the development of upperlimb non-invasive ambulatory robotic exoskeletons is presented as a promising solution for patients who cannot benefit from medication to suppress the tremor. In this area robotic exoskeletons have emerged, in the form of orthoses, to provide motor assistance and functional compensation to disabled people. An orthosis is a wearable device that acts in parallel to the affected limb. In the case of tremor management, the orthosis must apply a damping or inertial load to a selected set of limb articulations.

Recently, some studies demonstrated that exoskeletons could achieve a consistent $40 \%$ of tremor power reduction for all users, being able to attain a reduction ratio in the order of $80 \%$ tremor power in specific joints of users with severe tremor. In addition, the users reported that the exoskeleton did not affect their voluntary motion. These results indicate the feasibility of tremor suppression through biomechanical loading [4], [5].

The main drawbacks of this mechanical management of tremor are:

1) The resulting bulky solutions,

2) The inefficiency in transmitting loads from the exoskeleton to the human and musculo-skeletal system

3) Technological limitations in terms of actuator technologies.

In this regard, current trends in this field are focused on the evaluation of the concept of biomechanical loading of tremor through selective Functional Electrical Stimulation (FES) based on a (Brain-to-Computer Interaction) BCI-driven detection of involuntary (tremor) motor activity.

\section{BLOCK DIAGRAM AND DESCRIPTION}

Figure 1 shows the block diagram of the system for data assess. It includes PIC 16F877A microcontroller, sensors, alarms and LCD display. The detailed description of the same is given below.

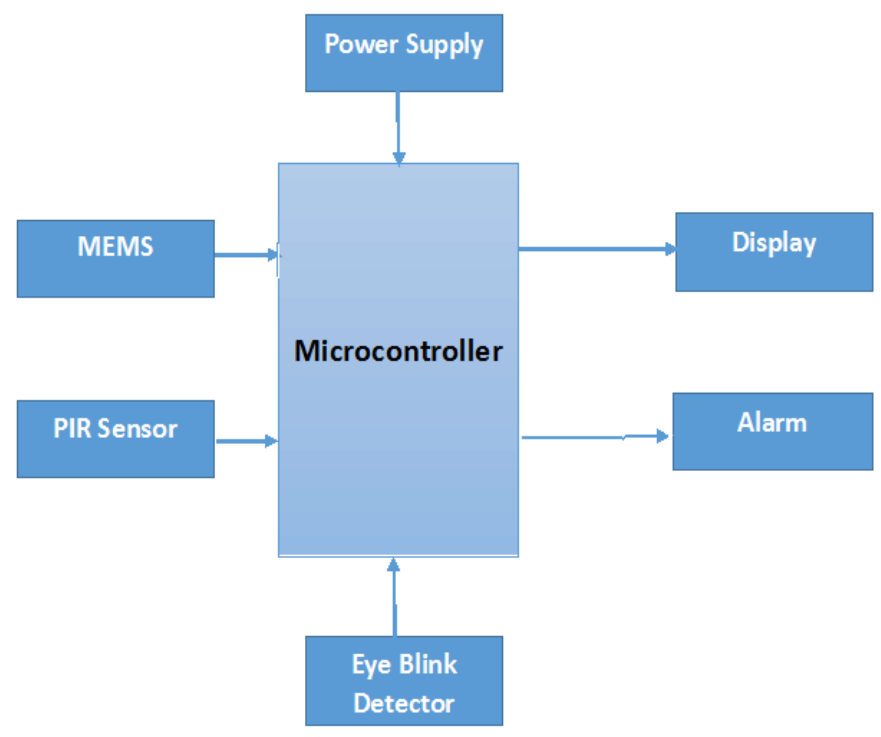

Figure 1: Block Diagram of the System for Data Assess

\section{A. PIC 16F877A}

PIC is a family of modified Harvard architecture microcontrollers made by Microchip Technology, derived from the PIC1650 originally developed by General Instrument's Microelectronics Division. The name PIC initially referred to "Peripheral Interface Controller". This is very small and easy to implement for non-complex problems.

The PIC 16F877A has following features

- High-performance RISC CPU

- Only 35 single word instructions to learn

- All single cycle instructions except for program Branches which are two cycle

- Operating speed: DC - $20 \mathrm{MHz}$ clock input

- Up to 8K x 14 words of FLASH Program Memory,

- Up to 368 x 8 bytes of Data Memory (RAM)

- Up to 256 x 8 bytes of EEPROM data memory

- Interrupt capability (up to 14 sources)

- $\quad$ Eight level deep hardware stack

- Direct, indirect and relative addressing modes

- $\quad$ Power-on Reset (POR)

- Power-up Timer (PWRT) and

- Oscillator Start-up Timer (OST)

\section{B. Sensors}

Three types of sensors are used in this implementation: Microelectro mechanical-system (MEMS) The MEM sensor we make use here is ADXL335 which is an accelerometer to reduce big mechanical Vibrations into small units. It is a small, thin, low power, complete 3-axis accelerometer with signal conditioned voltage outputs. It measures the static acceleration of gravity in tilt-sensing applications, as well as dynamic acceleration resulting from motion, shock, or vibration. The features of MEMS sensor are as follows:

- 3-axis sensing

- Small, low profile package

- $4 \mathrm{~mm} \times 4 \mathrm{~mm} \times 1.45 \mathrm{~mm}$ LFCSP

- Low power : $350 \mu \mathrm{A}$ (typical)

- Single-supply operation: $1.8 \mathrm{~V}$ to $3.6 \mathrm{~V}$

- $10,000 \mathrm{~g}$ shock survival

- BW adjustment with a single capacitor per axis

- RoHS/WEEE lead-free compliant

- Excellent temperature stability

1) Passive infra-red sensor (PIR)

It is an electronic device that measures infrared (IR) light radiating from objects in its field of view. It is made of Pyroelectric sensor which can detect levels of infrared radiation.

2) Eye blink detector

It is a long-range, infrared eye blink detector which also called an obstacle detector. It has a transmitter which transmits Infrared rays and the output of a receiver can be fed to a comparator (op-amp).

3) Alarm

We make use of Piezo buzzer which is an electronic device used to produce sound. It makes use of inverse principle of piezo-electricity. When a DC voltage is applied to the input pins, it is converted to an 
oscillating signal using resistor, transistor and inductor coil. This causes the metal plate to bend $\mathrm{n}$ opposite direction, produces sound waves in the air.

4) Display

A liquid crystal display (LCD) is used for displaying the result. LCD is a thin, flat display device made up of any number of colour or monochrome pixels arrayed in front of a light source or reflector. It is often utilized in battery-powered electronic devices because it uses very small amounts of electric power. This display structure is used widely for more than a few display elements.

\section{METHODOLOGY}

The MEMS inertial sensor placed at point of interest on the human body. The sensor converts the mechanical vibrations of body into electrical signals. Thus generated signals are fed to Port-A (pins A0, A1, A2) of Microcontroller. Port A is initialized to work as ADC which digitizes the analog signals from the MEMS. So digitized samples are then filtered through window of particular size and only interested samples are taken for further processing. Next selected samples are checked for periodicity. If samples show Pseudoperiodicity, it indicates the partial presence of Tremor.

The IR sensor's output is fed to Port B (pin B0) of Microcontroller. IR sensor senses the motion of any object present in front of it. IR sensor is used to count the number of eye blinks made by the person. If count goes beyond the threshold, it indicates the partial presence of the Cerebellar Tremor.

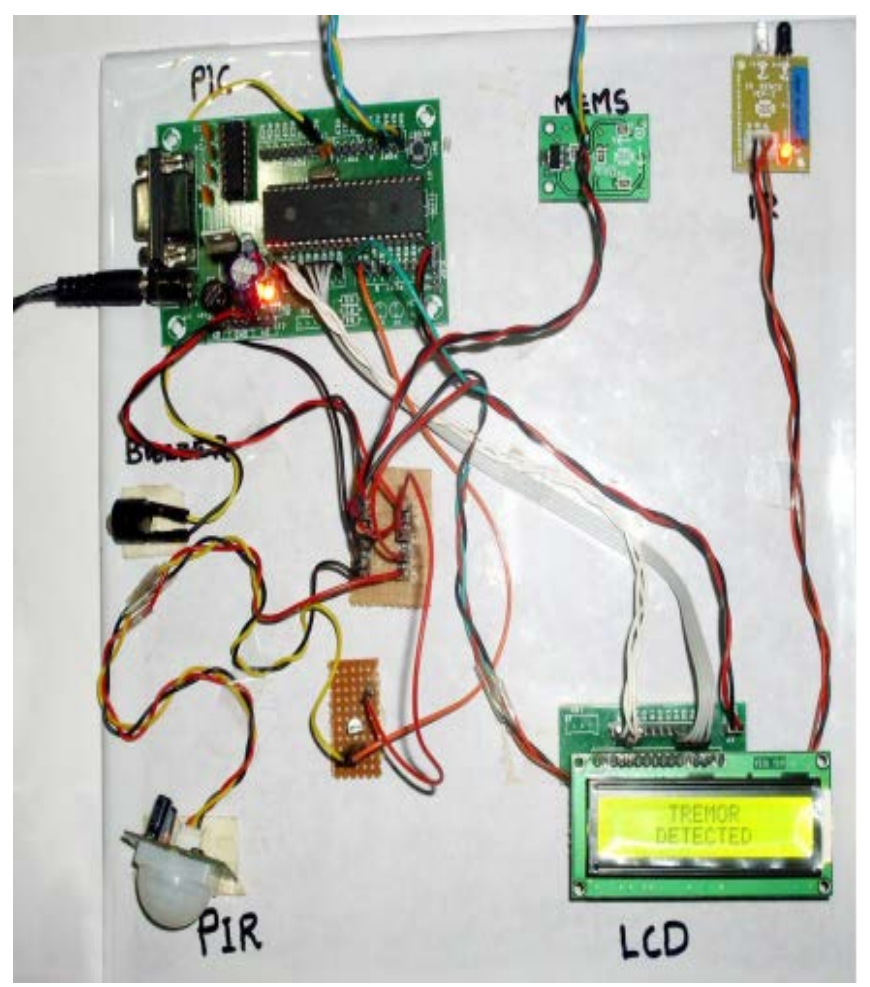

Figure 2: Complete Assessing Module of Tremor Detector
The PIR sensor's output is amplified and fed to Port B (pin B1) of Microcontroller. PIR sensor senses the change in infrared levels of the environment within its range. PIR is used to monitor the patient virtually when doctor is not around. PIR is so placed that patient will be in its focus. When patient will be lying still the output will be low, if patient moves the output of goes high. If output goes high, microcontroller senses a high signal at its pin B1 and it alarm the Buzzer. The complete assessing module of tremor detector is as shown in figure 2:

\section{CONCLUSION AND Future SCOPE}

\section{A. Conclusion}

This work describes the prototype-wearable inertial sensing technology. Hence, the designed Prototype meets the application requirements for clinical testing and addresses engineering constraints, such as low-power and resourceconstrained operation.

The paper also presents signal-processing techniques, such as digital filtering and sample comparing for periodicity that convert raw inertial data into clinically relevant, nonobvious information in the context of tremor assessment. While these and other techniques can be implemented on desktop computers for data post processing, many scenarios call for on-body processing, including applications requiring real-time assessments and energy-efficient solutions requiring selective data transmission and storage. Digital filtering is very simple and basic process in digital world. Further the future research work can include the use of special DS Processors along with some highly sophisticated algorithms.

\section{B. Results}

Parkinson tremor is detected by MEMS sensor. The MEMS Sensor gives the data samples which indicate the presence of tremor. The obtained data samples will be considered under two conditions. Then the presence of Tremor is displayed based on the following criteria:

i) With reference to the clinical data, the amplitude range of $60-100 \mathrm{mV}$ should be measured.

ii) 12th, 13th, 14th samples should have their amplitude equal to amplitude of $1^{\text {st }}$ sample with \pm 0.8 variations (Frequency check).

The presence and absence of tremor is determined in this process. The number of eye blinks made by the subject is counted using IR sensor. A preset condition is set for counting the number of times eyes are blinked. The preset is set in between 50 and 60 count of eye blinks to indicate the tremor is detected and displayed as C. The blank display indicates if number of eye blink goes beyond 60 .

The alarm is set for any movements of patient and it is checked by PIR sensor.

Case 1: Absence of Tremor

Samples acquired are:

5254586265586769726382785385828387835793 6893727873765570727482856587677855636975 8991999397 
Samples taken after first condition

6265676972638278858283878393689372787376

7072748285658767786369758991999397

$1^{\text {st }}$ sample 62

$12^{\text {th }}$ sample 87

$13^{\text {th }}$ sample 83

$14^{\text {th }}$ sample 93

Output on display is TREMOR NOT DETECTED

Case 2: Presence of Tremor

Samples acquired are:

6065745259637985899259658478879958646965

7888987970666974869491798269727687987566

679969758597

Samples taken after first condition:

6065746379858992658478879964696578889879

7066697486949179826972768798756667996975

8597

$1^{\text {st }}$ sample 60

$12^{\text {th }}$ sample 87

$13^{\text {th }}$ sample 60

$14^{\text {th }}$ sample 60

Output on display board displays as "TREMOR

DETECTED”.

\section{FUTURE SCOPE}

This work continues to improve the reliability of the prototype with real medical applications. Using suggestions from physicians and medical researchers related to the new concerns and criticality, the diagnosis and therapy are to be planned depending on the necessity of the requirements for treating movement disorders. Researches are carried out with optimal methods for making use of signal-processing algorithms. In addition to this work can be carried out in optimising the power consumption and wear ability. Better adaptive wireless communication techniques can be employed in improving the fidelity. Further, the system parameters can be measured and monitored to make use in telemedicine applications.

\section{REFERENCE}

[1] G. Deuschl, R. Wenzelburger, K. Loffler, J. Raethjen, and H. Stolze, "Essential tremor and cerebellar dysfunction," Brain, vol. 123, Pp. 1568-1580, 2000.

[2] S. Smaga, "Brain,” Amer. Family Phys., vol. 68, no. 8, Pp. 1546-1552, 2003.

[3] K. E. Lyons, R. Pahwa, K. L. Busenbark, A. I. Troster, S. Wilkinson, and W. C. Koller, "Improvements in daily functioning after deep brain stimulation of the thalamus for intractable tremor," Movement Disorders, vol. 13, no. 4, Pp. 690-692, 1998.

[4] H. C. Powell, Jr., M. A. Hanson, and J. Lach, "A wearable inertial sensing technology for clinical assessment of tremor," in Proc. IEEE Biomed. Circuits Syst. Conf., Pp. 9-12, 2007.

[5] E. J. W. van Someren, "Actigraphic monitoring of movement and restactivity rhythms in aging, Alzheimer's disease and Parkinson's disease," pp.394-398,Rehabilitation Engineering, IEEE Transactions on Vol: 5, Issue: 4, Dec-1997 